\title{
Calcium imaging in a FlAsH
}

A new calcium indicator called Calcium Green FlAsH allows researchers to monitor rapid and localized calcium dynamics in the cell.

Got calcium? It's not just for strong bones. Calcium is an important second messenger in cell signaling, carrying the weighty responsibility of regulating rapid functions such as muscle contraction and neuron firing.

In neurons, calcium has a tendency to build up in 'microdomains' around the mouths of calcium channels, poised and ready to be rapidly dispatched as a signal. Roger Tsien of the University of California at San Diego and his colleagues have now developed a method to image rapid and localized calcium dynamics in the cell, with the design of a new fluorescent small-molecule indicator they call Calcium Green FlAsH (CaGF).

Several small-molecule fluorescent calcium indicators are available that allow the bulk measurement of calcium in a cell. As first author Oded Tour explains, however, "calcium concentration inside cells may vary greatly both spatially and temporally, [which] could have a profound effect on the physiological signal that calcium is conveying." Genetically encoded calcium indicators can be attached to a target protein, allowing localized imaging. But these protein-based indicators have slow responses, making it difficult to monitor fast calcium dynamics, and their bulky girth can disrupt cellular function.

CaGF combines the advantage of smallmolecule indicators in that its fluorescent response is very rapid, with the benefit of genetically encoded indicators in that it can be targeted to a protein of interest. This unique probe, based on Tsien's FlAsH biarsenical dye scaffold, was designed and synthesized by chemist Stephen Adams. It contains three chemical moieties (Fig. 1a): a $\mathrm{Ca}^{2+}$ chelator, a fluorescein dye and two arsenic atoms that form four covalent bonds to proteins tagged with a tetracysteine motif. The fluorescence of CaGF increases dramatically upon binding calcium. Notably, its affinity for calcium is relatively weak, which Adams explains: "Low affinity results in fast off-rates of calcium from the indicator, allowing submillisecond changes to be measured."

To demonstrate that CaGF could report on calcium dynamics in living cells, Tsien, Tour and their colleagues tagged recombinant connexin 43 with a tetracysteine motif in HeLa a

a

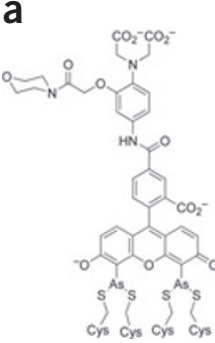

b

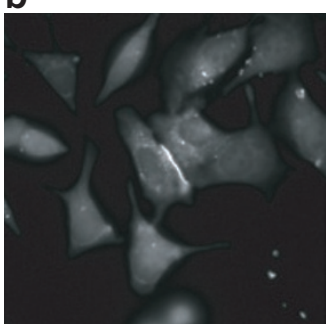

Figure 1 | Calcium imaging with CaGF. (a) The CaGF indicator consists of a $\mathrm{Ca}^{2+}$ chelator (top), a fluorescein dye (middle) and two arsenic atoms that bind a tetracysteine tag on a protein (bottom). (b) CaGF reports a large gap junction between two HeLa cells expressing tetracysteinetagged connexin 43. Reprinted from Nature Chemical Biology.

cells. They observed intense fluorescence at gap junctions (Fig. 1b), where connexin 43 is known to localize.

The researchers next tagged an L-type calcium channel in HEK293 cells, which, owing to its diffuse expression pattern, is much more difficult to image than connexin 43 . They imaged elevated calcium levels upon depolarization of the membrane by patch clamping. Unexpectedly, they observed 'hot spots' of fluorescence, which indicated dense microdomains of active channels, while most calcium channels were closed and did not transport calcium. Though calcium channels appeared to be uniformly distributed at the cell membrane, the researchers concluded that hot spots arise as a result of the nonuniform distribution of responsive channels, something that could not have been observed with the previous generation of calcium indicators.

Tsien and his colleagues' interesting findings as well as the useful properties of $\mathrm{CaGF}$ should generate excitement in the field as a tool for imaging rapid and local calcium dynamics in the cell. Furthermore, the successful design of a genetically targeted smallmolecule indicator based on the FlAsH scaffold could serve as a model for building others, as Tour explains, "indicators for other small-molecule second messengers could benefit from this approach ... providing [that they] can be made cell-permeable."

\section{Allison Doerr}

\section{RESEARCH PAPERS}

Tour, 0. et al. Calcium Green FlAsH as a genetically targeted small-molecule calcium indicator. Nat. Chem. Biol. 3, 423-431 (2007). 